\title{
Téoros
}

Revue de recherche en tourisme

\section{Entrevue avec le politicologue Jacques Léveillé}

\section{Jean Pelletier}

Volume 9, numéro 1, mars 1990

Plans et politiques touristiques

URI : https://id.erudit.org/iderudit/1080285ar

DOI : https://doi.org/10.7202/1080285ar

Aller au sommaire du numéro

Éditeur(s)

Université du Québec à Montréal

ISSN

0712-8657 (imprimé)

1923-2705 (numérique)

Découvrir la revue

Citer ce document

Pelletier, J. (1990). Entrevue avec le politicologue Jacques Léveillé. Téoros, 9(1), 8-10. https://doi.org/10.7202/1080285ar d'utilisation que vous pouvez consulter en ligne.

https://apropos.erudit.org/fr/usagers/politique-dutilisation/ 
En jetant un regard sur l'évolution des rôles de l'État (fédéral, québécois et municipal) au fil des trois ou quatre dernières décennies, peut-on en brosser un portrait et en tirer quelques enseignements?

Le portrait que l'on peut tracer du rôle, des actions et des influences de l'État fédéral et de l'État provincial québécois depuis la fin de la Deuxième Guerre Mondiale est relativement bien connu. D'une situation d'État minimal, soit un État qui laisse à la société civile le soin de gérer elle-même un ensemble de questions (santé, école, loisirs, culture), on est progressivement passe à un État-providence, soit un État qui s'attribue une responsabilité sur pratiquement toutes les dimensions de la vie sociale. Depuis une dizaine d'années, cet État interventionniste est remis en question sous les pressions de la société civile, pressions qui trouvent des échos favorables au sein des appareils étatiques par suite des difficultés de financement des rôles que l'État-providence s'était accaparés. Certains diront que nous sommes entrés dans une phase de recherche d'un nouveau contrat entre la société civile et la société politique.

Au plan municipal, le même cheminement peut être observé quoique les phases soient décalées. Ceci tient au fait que les municipalités évoluent à l'intérieur d'une double contrainte: elles gèrent des pouvoirs délégués d'une part, et elles ont l'obligation de confectionner des budgets equilibrés. N'ayant ni le loisir d'étendre leurs champs d'intervention sans permission du gouvernement provincial, ni la capacité d'accumuler des déficits comme au fédéral et au provincial, les municipalités ont été plus lentes à s'engager sur la voie de l'État-providence. Il aura fallu attendre le désengagement des autres niveaux degouvernement pourqueles municipalités, et en particulier les villes, décident d'occuper des domaines d'intervention moins traditionnels. Ce décalage dans l'émergence d'un État-providence local n'a toutefois pas empêché que la remise en question de l'État-providence s'adresse aussi bien à l'État local qu'aux autres niveaux de l'appareil étatique.

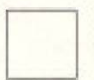

En quoi le cheminement québécois se distingue-t-il de l'évolution observée à l'échelle canadienne?

La comparaison d'expériences sociales, politiques et gouvernementales n'est pas toujours facile. L' histoire des relations entre la société́ civile et la société politique joue un grand rôle dans la rapidité et dans la nature des étapes que nous venons de tracer. Il y a des sociétés où la société civile a pratiquement toujours été sous le contrôle, ou même la tutelle de l'État. II y a d'autres sociétés où la tradition décentralisatrice a été plus importante et où la société civile a cherché à maintenir des mécanismes de résistance à la présence envahissante de l'État. Ceci ne veut pas direque l'État n'est pas intervenu. Par contre, les séquences de l'intervention ont été différentes dues à la tradition.

Le Québec, plus que le Canada et que les autres provinces, $s$ 'est inspiré de l'une et l'autre tradition pour rếgir ses rapports entre la société civile et la société politique. Ainsi, la tradition décentralisatrice rend bien compte de l'évolution du Québec jusqu'à la fin de la Deuxième Guerre Mondiale, et même jusqu' aux débuts des années soixante. Par contre, avec l'avènement de la Révolution Tranquille, la tradition étatique a pratiquement occupé toute la place. En l'espace de quelques années, la société québécoise s'est laissée convaincre de la nécessité de la planification, de l'utilité de la prise de contrôle, par l'État, d'une foule de domaines d'intervention, de l'urgence, pour l'État de se transformer en entrepreneur, etc.. Bref, si on identifie la tradition décentralisatrice aux expériences anglosaxonnes et la tradition étatique à l'expérience française, nous pouvons dire que le Québec est rapidement passé à un mode de gestion à la française après 1960 . Ces illustrations d"un tel passage sont nombreuses: intention de créer un ministère du Plan, rationalisation des instances de décentralisation dans le domaine de la
Monsieur Jacques Léveillé est prolesseur su dépara Montreal 
santé, de l'éducation, des municipalités, création d'organismes para-gouvernementaux èt de sociétés d'État.

On a souvent eu tendance à parler de l'État québécois des années '60 et ${ }^{\prime} 70$ comme d'un État-providence. Depuis une dizaine d'années, on observe une nette volonté de réduire sensiblement son rayonnement. Qu'en est-il exactement?

La notiond'État-providence recouvre l'idée d'une invasion de l'État ou d'une présence tutélaire de l'appareil étatique. On dit alors. que l'État prend les gens en charge de la naissance à la mort afin que ces demiers soient de moins en moins soumis aux aléas. et aux désordres d'une société et d'un marché libres. Or, cette protection ne se réalise pas sans le développement de moyens de prévision, de planification et d'organisation de l'avenir. Tout comme dans notre vie personnelle, la volonté de réduire le nombre de surprises désagréables exige que nous prévoyions les étapes, que nous planifions les ressources en vue de répondre à des imprévus ou pour réaliser certains projets de voyage ou de retraite, de même en va-t-il d'un État qui se reconnaît un rôle de père et de mère à l'égard de ses enfants-citoyens.

La logique d'evolution del'État-providence implique la montée d'une expertise technique et professionnelle. Pour sa part, la logique de l'expertise est telle que les expressions spontanées et intuitives du monde ordinaire sont inévitablement dépréciées lorsque comparées à ce que transmet laconnaissance scientifique des techniciens et des experts. Les ingénieurs sociaux se transforment en bureaucrates étatiques au sein de l'État-providence. Ils cherchent également à transposer cette même logique à divers niveaux de la société civile. Jusqu'à un certain seuil de tolérance de la part de la société civile.

Votre question contient l'idée d'une décélération rapide de cette mentalité et de cette logique associées à l'État-providence. Estce vraiment aussi rapide? II importerait d'en faire une analyse rigoureuse. Il est peut-être plus facile de cemer les raisons et les effets de la décélération que l'on observe dans toutes les sociétés occidentales.

Les tenants du néo-libéralisme et les théoriciens des biens publics (public choice) sont probablement les critiques les plus articulés et les plus virulents de l'Etatprovidence. Ils soutiennent que l'État en donne plus que le client en demande ou en a besoin. Ils ajoutent que les bureaucraties gouvemementales et para-gouvernementales sont parvenues au stade où elles s'alimentent et se développent pour ellesmêmes et par elles-mêmes. Ils concluent que l'ajout de moyens financierset humains dans ces organisations ne doit être encouragé sous aucun prétexte puisqu'au lieu d'aider à la solution des problèmes sociaux et économiques, la croissance de l'appareil étatique décourage les initiatives privées et déresponsabilise l'ensemble des intervenants. A l'aide d'exemples puisés dans divers secteurs de l'intervention bureaucratique, ils proposent un retrait substantiel de l'État et un ré́quilibrage des rapports entre société civile et société politique. Les plus radicaux vont jusqu'à revendiquer un passage du tout-à-1'État au tout-à-la-société-civile.

Le renversement de tendances n'est-il pas dû d'abord aux contraintes d'ordre budgétaire plutôt qu'au renouvellement des valeurs?

Il y a effectivement une convergence des forces. D'un cốté, l'État a pris en charge un ensemble de programmes sociaux qui cô̂tent très chers et dont les ramifications sont pratiquement sans fin. Aussi, les déficits budgétaires agissent comme des signaux d'alarme en égard à la capacité de redistribuer la richesse collective. D'un autre côté, certains intervenants socio-économiques ont noté que les argents soutirés par l'État à des fins de redistribution diminuaient d'autant les capacités d'investir dans la création de nouvelles richesses. D'où une convergence des pressions pour que l'État libère les ressources des particuliers et des corporations pour relancer un cycle de production de richesses.

Sur un plan plus global, vous pouvez mẻme dire que cette convergence des forces ne concerne pas que les anti-étatiques et les milieux $\mathrm{d}^{\dagger}$ affaires, mais également les socialistes, les communautaristes et les anarchistes qui exigent désormais un dégraissage de l'État pour donner une chance à l'expression décentralisée des besoins et des solutions.

\section{Dans cette perspective,} n'assistons-nous pas à l'émergence d'un "nouveau contrat social" aux allures universelles?

Les exemples récents des pays de l'Est sont révélateurs de cette tendance à l'échelle internationale. En effet, des régimes très providentiels ont été contestés par des forces émanant de la société civile pour que la présence de l'État soit moins envahissante et pour que la société ait une chance de libérer ses énergies. En peu de mois, nous avons pris conscience que les parties prenantes à un nouveau contrat étaient très nombreuses et parfois très articulées.

En pensant à l'avenir immédiat les dix prochaines années -, quels seront les champs d'intervention qui continueront d'être occupés par les gouvernements d'ici?

Vous soulevez d'immenses questions. Il est permis de penserque les États de l'avenir délaisseront leur rôle d'entrepreneur pour mieux se consacrer à leur fonction de superviseur de la vie en société. Ainsi, pour accomplir un retour complet sur l'État minimal, les États définiront les grandes orientations et les grands objectifs à atteindre sans se reconnaître l'obligation de gérer les moyens d'y parvenir. Les formes d'accompagnement ou d'assistance aux initiatives de la société civile seront évidemment diversifiées et adaptées aux traditions dont nous avons parlé plus haut. Déjà nous voyons poindre, à divers niveaux de gouvernement, des expériences de partenariat, de co-gestion, ou mềme de délégation de responsabilités.

La Ville de Montréal constitue, sous cet angle, un beau cas d'étude. En dépit du fait que l'administration du RCM ait été identifiée au courant social-démocrate, la pratique observéedans divers secteurs n'est pas une pratique interventionniste, mais plutôt une pratique d'accompagnement. Ainsi, dans le secteur du tourisme par exemple, l'administration ne semble pas avoir cherché à exercer un contrôle interventionniste après avoir contribué à rassembler les énergies qui désiraient s'exprimer. Elle a consenti à fusionner ses moyens d'intervention à ceux des intervenants privés sans réclamer le leadership des efforts consentis.

\section{Allons-nous vraiment passer de l'État pourvoyeur et omniprésent à un État minimaliste?}

Votre question exige une double réponse. D'une part, nous cherchons à saisir une situation de transition entre un Étatprovidence interventionniste, et même entrepreneur, et un État superviseur et accompagnateur. II est inévitable que les deux mondes co-existent pendant un temps. D'autre part, il est prévisible que l'État persistera dans son rôle d' intervenant principal dans divers domaines. Il est d'ailleurs essentiel qu'il en soit ainsi pour que 
1'appareil étatique ait une emprise concrète sur les réalités et pour que le secteur public constitue un secteur témoin de première importance.

La mise en place de nouvelles formes de rapports sera probablement plus rapide au niveau local. Ceci est dû au fait que, dans nos sociétés, le gouvernement ou l'administration locale est souvent perçu comme une instance de la société civile, tout autant qu'un maillon de l'appareil étatique. Ainsi, lorsque les réformateurs de l'État-providence prónent un retour aux collectivités locales, le gouvemement local tend à être identifié à un corps intermédiaire, à une association volontaire ou à un groupe d'intervention. Selon cette vision des choses, l'administration locale n'est pas très différente de la Chambre de com-

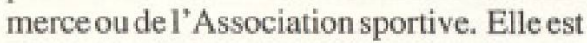
simplement plus multi-fonctionnelle, du moins en théorie. Dans certaines municipalités, l'ensemble de ces regroupements locaux partagent les mêmes locaux, se distribuent les interventions sans que la distinction entre collectivité locale publique et collectivité locale privée ne soit tranchée. Cette fusion de la société civile et de la société politique, au niveau local, permet de réaliser plus facilement les modalités d'expressiondunouveau contrat social dont nous avons parle. Le réflexe de tout confier à l'État n'ayant pas eu le temps des' incruster au niveau local, comme cela s'est produit aux autres niveaux de gouvernement, le partenariat, la co-gestion, la privatisation, etc., ne sont pas perçus comme des solutions contre-nature. De plus, dans la grande majorité des cas, les administrations locales sont incapables de prendre sur elles de gérer un grand nombre de services ou de s'emparer de multiples responsabilités. II fallait s'en remettre aux initiatives communautaires et aux entreprises privées.

Au niveau du gouvernement du Québec, la situation est sensiblement différente. L'appareil étatique a été beaucoup plus envahissant et beaucoup plus entreprenant. Ayant aspiré vers lui un ensemble de responsabilités autrefois assumées par les collectivités locales, il s'est donné des moyens substantiels pour composer la musique et diriger l'orchestre. Le délestage ne pourra être que plus lent. Les forces qui soutiennent la continuité d'une présence étatique élaborée et diversifiée sont capables de montrer une résistance aux forces qui revendiquent un dégraissage substantiel de $l^{\dagger} E$ tat provincial.
L'évolution des institutions étatiques qui sont les nôtres va-telle se poursuivre à des rythmes différents?

Il est permis de penser que la tendance s"exprimera de façon différente au Québec parce que la société québécoise est effectivement distincte. A cause de son histoire et de sa position en Amérique du Nord, le Québec est une société très solidaire, une société qui se serre assez rapidement les coudes devant certaines adversités ou face à certains défis. La conjoncture actuelle offre plusieurs illustrations de cette solidarité qui s' articule parfois avec l'État et parfois sans l'État ou à l'extérieur de l'Etat. Bref, l'État n'est pas, pour la majorité de la population québécoise, un instrument de destruction de solidarité sociale. Pour plusieurs dimensions fondamentales de son devenir, la société québécoise ne semble pas vouloir un retrait de l'État. Il y a donc ici une solidarité avec l'État qui est différente de la solidarité exprimée dans des sociétés où le sentiment d'appartenance et l'affection nationale sont moins vifs.

Comment l'observateur-chercheur que vous êtes situe-t-il les interventions publiques dans le domaine récréotouristique?

En effectuant des recherches sur la réforme municipale au Québec, j'ai toujours été surpris par le nombre de groupes et association d'interventionqui s'exprimaient et qui se réalisaient en dehors de toute structure gouvernementale avant que la réforme municipale ou régionale ne s'intéresse à eux. Par exemple, dans le domaine des loisirs et du tourisme. Est-ce que l'insertion de ces groupes et associations dans les structures consultatives et autres ont fait avancer les questions qu'ils tentaient de faire progresser sans encadrement gouvernemental? Est-ce que cette récupération, au moins partielle, des initiatives privées par l'appareil étatique aeu une influence positive ou négative sur le dynamisme initial? Je nesaurais être catégorique. Par ailleurs, il semble bien que dans le domaine du loisir et du tourisme, les intervenants communautaires ont beaucoup plus besoin d'un support à leurs initiatives que $\mathrm{d}^{\dagger} u n$ encadrement normatif et tutélaire de leurs actions. Plutôt que de tenter de niveler les expériences, il importe d'encourager l'émergence d'un grand éventail de choix et de respecter ceux et celles qui acceptent de promouvoir ces choix par intérêt. A trop vouloir uniformiser, l'État risque de décourager les initiatives ou de reproduire, dans

10 TÉOROS-Wd. I,m. I, mars 190 les groupes privés, les travers bureaucratiques de l'appareil étatique.

La première tâche des intervenants publics consiste à bien connaître le milieu dans lequel ils projettent une intervention. Il ne suffit pas de constater que les entreprises et associations oeuvrant dans le secteur du tourismesont très nombreuses pour conclure qu'il faille les regrouper, d'une façon ou d'une autre, en utilisant l'argument qu'elles visent à atteindre des buts objectivement convergents. En cherchant à savoir pourquoi elles ne se sont pas regroupés, il sera probablement possible de prendre conscience que ce serait une bêtise de vouloir les faire travailler ensemble. Certains de ces intervenants sont peut-être incapables de se voir, même en peinture. Aussi, la pire solution serait la solution purement bureaucratique.

Vaut probablement mieux recourir à une stratégie plus politique dans laquelle l'autorité publique, qui en a la légitimité, $s^{*}$ introduit dans tous les groupes sans s'identifier à l'un ou à l'autre. Certes, les résultats sont plus longs à se manifester, mais il n'y a souvent pas d'autres choix. Bref, plutôt que de croire à la transposition des solutions d'un secteur à l'autre, il importe de concevoir des stratégies diversifiées sur la base d'une connaissance intime des milieux et des intérêts qui mobilisent les divers intervenants. Au terme d'un tel processus, l'autorité publique ne devrait pas hésiter à prendre le leadership, ou à jouer un rôle de second plan, si tel est la conclusion la plus efficace. Il semble bien que la Ville de Montréal ait adopté cette deuxième solution dans le secteur du tourisme à Montréal.

En vous écoutant, on en vient à souhaiter que les gouvernements créent des incubateurs d'idées et de projets...

Tout à fait. Les villes sont d'ailleurs à se doter de tels instruments de réflexion. Ici comme ailleurs, les villes prennent conscience que le XXIe siècle les propulsera sur le devant de la scène dans de nouveaux secteurs d'intervention comme le tourisme, la culture ou l'environnement. Or, par tradition, et peut-être aussi par nécessité, les villes et les instances régionales sont plus ouvertes à des modalités d'intervention qui réalisent une synthèse de la société civile et de la société politique. Reste à savoir si les appareils étatiques qui s'ellaborent au niveaudes villeset des instances régionales actualiseront avec bonheur ce potentiel innovateur dont elles bénéficient, du moins en theorie. $f$ 